\title{
Multiple Antennas and Representation Theory
}

\author{
Babak Hassibi, Bertrand Hochwald, Amin Shokrollahi, and Wim Sweldens \\ Mathematical Sciences Center \\ Lucent Technology \\ 600 Mountain Avenue \\ Murray Hill, NJ 07974 \\ e-mail: \{hassibi, hochwald, mshokrollahi,wim\}@lucent.com
}

\begin{abstract}
Multiple antennas can greatly.increase the data rate and reliability of a wireless communication link in a fading environment, but the practical success of using multiple antennas depends crucially on our ability to design high-rate space-time constellations with low encoding and decoding complexity. It has been shown that full transmitter diversity, where the constellation is a set of unitary matrices whose differences have nonzero determinant, is a desirable property for good performance.

We use the powerful theory of fixed-point-free groups and their representations to design high-rate constellations with full diversity. Furthermore, we thereby classify all full-diversity constellations that form a group, for all rates and numbers of transmitter antennas. The group structure makes the constellations especially suitable for differential modulation and low-complexity decoding algorithms.
\end{abstract}

The classification also reveals that the number of different group-structures with full diversity is very limited when the number of transmitter antennas is large and odd. We therefore also consider extensions of the constellation designs to nongroups. We conclude by showing that many of our designed constellations perform excellently on both simulated and real wireless channels.

A complete copy of this paper is available on the web at http://mars.bell-labs.com under the title "Representation Theory for High-Rate MultipleAntenna Code Design." Other related papers are also available at this web site.

\section{An Example of a High-Rate Code}

As an example of a high-rate group code that we find, we plot the performance of $\mathrm{SL}_{2}\left(\mathbb{F}_{5}\right)$, the group of $2 \times 2$ matrices over the field $\mathbb{F}_{5}$ with determinant one. This group has a representation as 120 complex $2 \times 2$ unitary matrices suitable for transmission over a two-antenna fading channel. The group is fixed-point-free which means that its constellation has full diversity. We also plot the performance of the best cyclic group with the same rate [2], a $2 \times 2$ orthogonal design [4] (which is not a group) and a generalized quaternion group code [3] with similar rates. All of these codes can be used with a known channel (as shown), or they can be used differentially when the channel is unknown and with a performance loss of approximately $3 \mathrm{~dB}$.

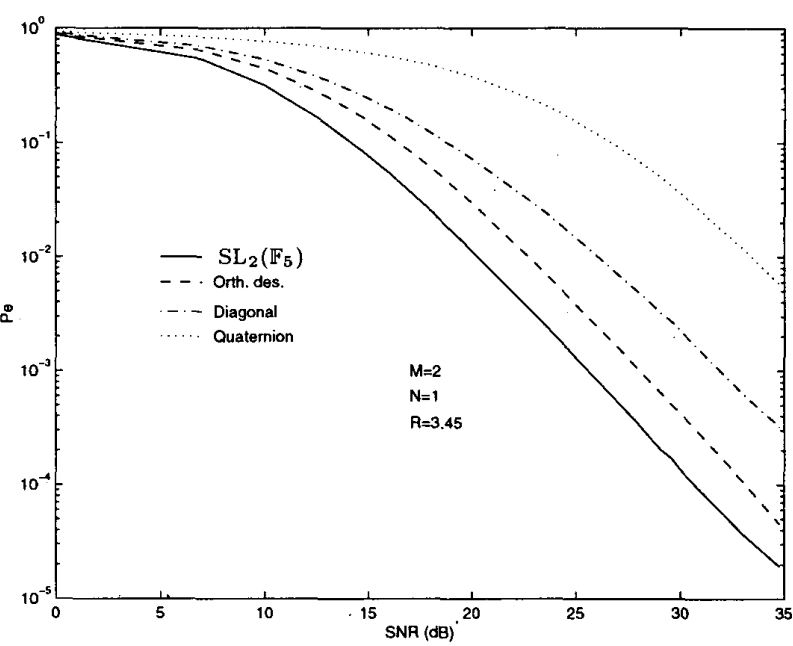

Block-error rate performance of the group $\mathrm{SL}_{2}\left(\mathbb{F}_{5}\right)$ compared with constellations from other constructions for $M=2$ transmitter antennas and $N=1$ receiver antenna. The channel is known at the receiver. The solid line is $\mathrm{SL}_{2}\left(\mathbb{F}_{5}\right)$, which has 120 unitary matrices (rate $R \approx 3.45$ ). The dashed line is an orthogonal design with 11 th roots of unity $(R \approx 3.46)$. The dashed-dotted line is the best diagonal (Abelian group) construction $(R \approx 3.45)$. The dotted line is the quaternion group with 128 matrices $(R=3.5)$.

\section{REFERENCES}

[1] B. Hassibi, B. Hochwald, A. Shokrollahi and W. Sweldens, "Representation theory for high-rate multiple-antenna code design," Bell Labs. tech. report, Mar. 2000.

[2] B. Hochwald and W. Sweldens, "Differential unitary space-time modulation," submitted to IEEE Trans. Comm., Also, Bell Labs. tech. report, Mar., 1999.

[3] B. Hughes, "Differential Space-Time Modulation," submitted to IEEE Trans. Info. Theory, 1999.

[4] V. Tarokh, H. Jafarkhani and A. R. Calderbank, "Space-time block codes from orthogonal designs," IEEE Trans Info. Theory, vol. 45, pp. 1456-1467, July 1999. 